\title{
Multi-physics and particle methods
}

\author{
Georges-Henri Cottet*
}

LMC-IMAG Université Joseph Fourier, Grenoble, BP 53, F-38041, France

\begin{abstract}
We present in this papers some concepts and results concerning the application of particle methods to complex physics. We focus on two examples involving interfaces: variable density flows with surface tension and a one-dimensional fluid-structure model.
\end{abstract}

Keywords: Interfaces; Particle methods; Fluid-structure interaction

\section{Introduction}

Particle methods are numerical tools which combine robustness and accuracy for the simulation of advectiondominated phenomena. They consist in tracking advected quantities with local mollified velocity. This velocity field can for instance be computed on a grid, leading to the so-called Particle-In-Cell (PIC) methods. When dealing with the 3D incompressible Navier-Stokes equations, it is economical to use this strategy as it allows to use gridbased Poisson solvers which are faster than summation formulas.

A typical PIC code consists in pushing particles, interpolate fields on a grid, use centered formulas to differentiate on the grid all quantities that are not directly related to the transport, and finally interpolate back to particles these quantities. Frequent regriding with a cubic spline interpolation kernel allows to maintain the regularity of the particle distribution which is crucial for accuracy. Fig. 1 is an example of a PIC calculation for isentropic gas dynamics. In this case the variables carried by particles are momentum, density and total energy. It shows a shock wave, reflected by a vertical wall and interacting with the boundary layer it has created along an horizontal wall. These results in good agreement with those obtained by up-to-date TVD finite-difference schemes using the same number of points [1]. In the case of incompressible flows, comparisons with spectral Direct Numerical Simulation results [2] point to the same conclusion. This paper focuses on the application of the method to the simulation of systems which combine different physical models; first a variable density flow, then a case of fluid-structure interaction.

\footnotetext{
* Tel.: +33 (4) 7651-4992; Fax: +33 (4) 7663-1263; E-mail: Georges-Henri.Cottet@imag.fr
}

\section{Variable density flows}

We consider an incompressible flow with variable density and surface tension. A level set approach [3] allows to formulate the problem as a system of advection equation for the vorticity $\omega$, the density gradient $\nabla \rho$ and a level function $\phi$ carrying the surface tension:

$$
\begin{aligned}
& \frac{\partial \omega}{\partial t}+\operatorname{div}(u \omega)=\mu \Delta \omega+\frac{\nabla p \times \nabla \rho}{\rho^{2}}+\tau \nabla \phi \times \nabla\left(\kappa(\phi) \zeta_{\epsilon} / \rho\right) \\
& \frac{\partial(\nabla \rho)}{\partial t}+\operatorname{div}(u \nabla \rho)+[\nabla u]^{t} \nabla \rho=0
\end{aligned}
$$

In the above system, $\kappa$ is the surface tension and $\zeta_{\epsilon}$ is a cutoff normalized at each point by the norm of the gradient of the level function $\phi$. The curvature, pressure gradient and density are computed on a grid after interpolation of the quantities carried by the particles. Fig. 2 shows the results of a simulation of the merger of two rising bubbles having different density (lighter fluid below) with (right picture) and without (left picture) surface tension (density ratio is 50 , viscosity is 0.0025 and surface tension is 0.05 ). These results are in very good agreement with the calculations reported in [3] with an ENO scheme using the same number of points.

\section{Fluid-structure interaction}

Let us consider the simple one dimensional fluidstructure model, coupling a Burger's equation in the fluid and linear elasticity in the solid:

$$
\begin{gathered}
\frac{\partial u}{\partial t}+\frac{3}{2} u \frac{\partial u}{\partial x}-v \frac{\partial^{2} u}{\partial x^{2}}=0 \quad \text { for } x \in[0, \gamma(t)], \\
\frac{\partial^{2} d}{\partial t^{2}}-\mu \frac{\partial^{2} d}{\partial \xi^{2}}=0 \quad \text { for } \xi \in[1,2]
\end{gathered}
$$




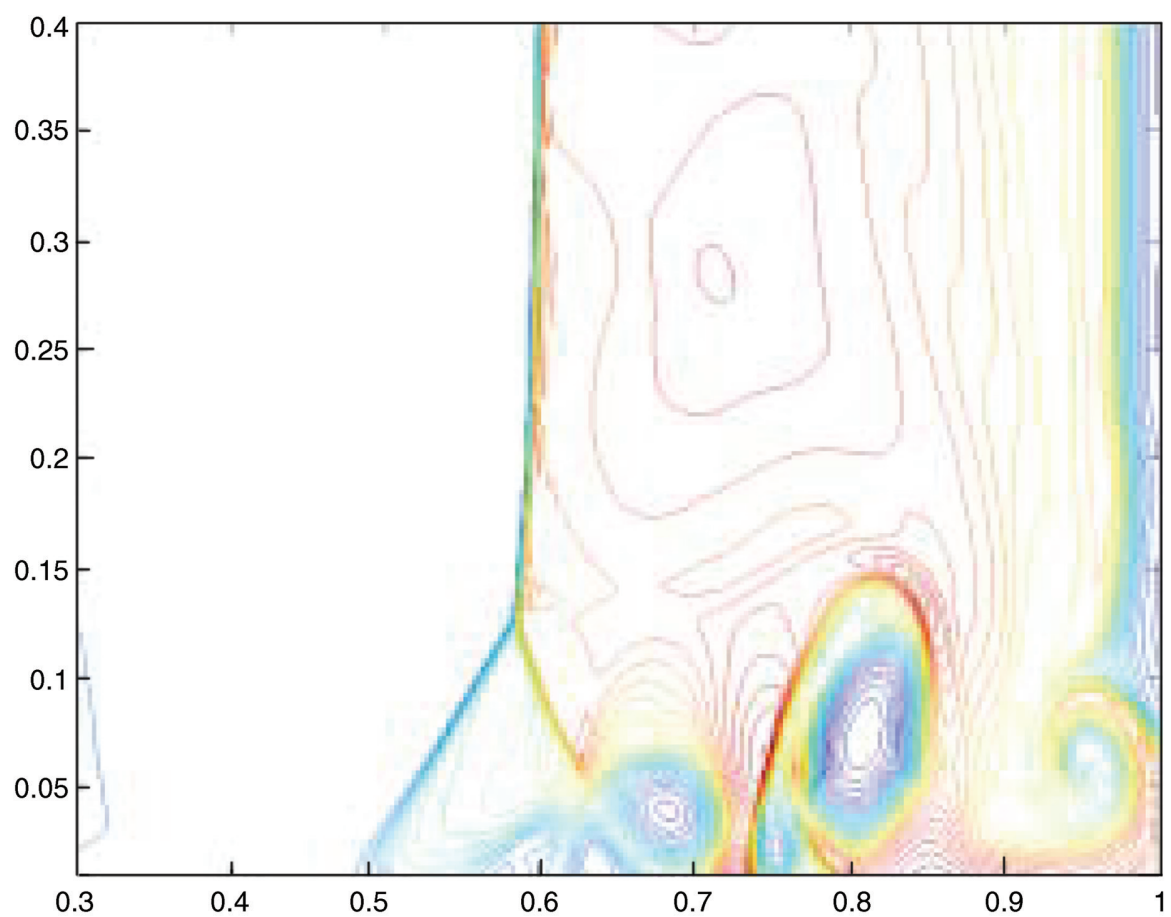

Fig. 1. Density contours of a shock-boundary layer interaction computed by a PIC method.

In the above equation $u$ is the velocity in the fluid phase, $d$ is the solid displacement. $x$ and $\xi$ are respectively the Euler and Lagrange coordinates and $\gamma(t)$ is the moving interface. The continuity of stresses at the interface $\xi=1$ reads

$v \frac{\partial u}{\partial x}(\gamma(t), t)=\mu \frac{\partial d}{\partial \xi}(1, t)$

One may observe that the wave equation for $d$ gives a non-linear Burger's equation in the Eulerian coordinate for $u$ in the solid. Moreover, the continuity of stresses allows to build a unique second order operator valid across the interface. These remarks lead to the following model

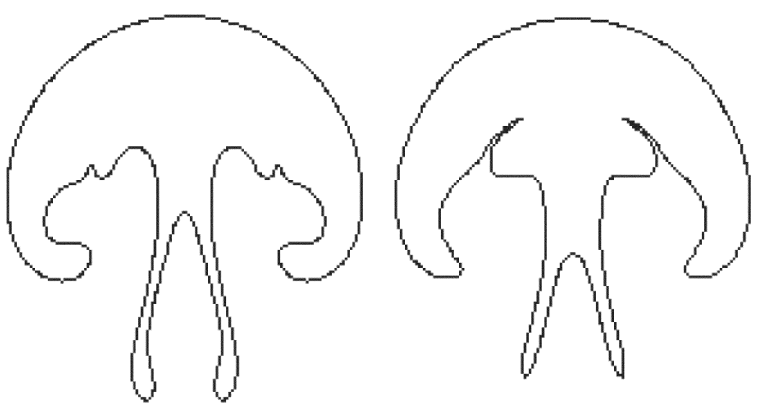

Fig. 2. Merger of 2 bubbles with density ratio of 50, with (right picture) or without (left picture) surface tension. involving solid (S) and fluid (F) particles:

$u(x, t) \simeq \sum_{p} u_{p} v_{p} \delta\left(x-x_{p}\right) ; \quad \dot{x}_{p}=U_{p}$,

$\dot{v}_{p}=U_{p}^{\prime} v_{p}, \quad \dot{u}_{p}=-U_{p}^{\prime} u_{p} \chi_{p}^{F} / 2+D_{p}$

where $\chi^{F, S}$ stand for the characteristic function of the fluid and solid phases, respectively, and

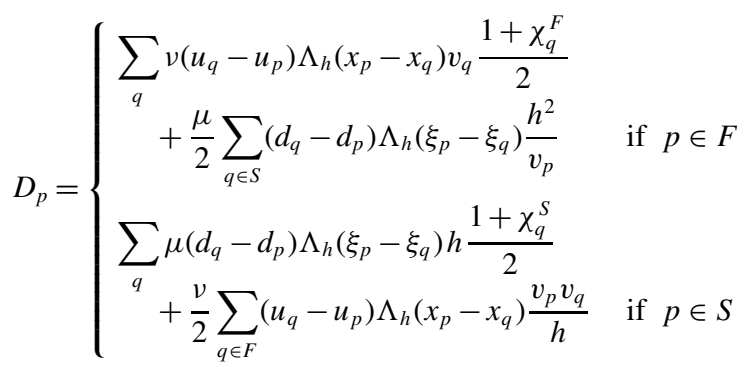

A remarkable feature of this model is that it satisfies the right energy inequality. Details can be found in [4]. Fig. 3 (left picture) shows a comparison of the results given by the particle model and a classical ALE model based on second order centered finite differences. The initial condition was a cosine velocity profile pushing the fluid to the left. The right picture summarizes a refinement study exhibiting an order of convergence between one and two. 

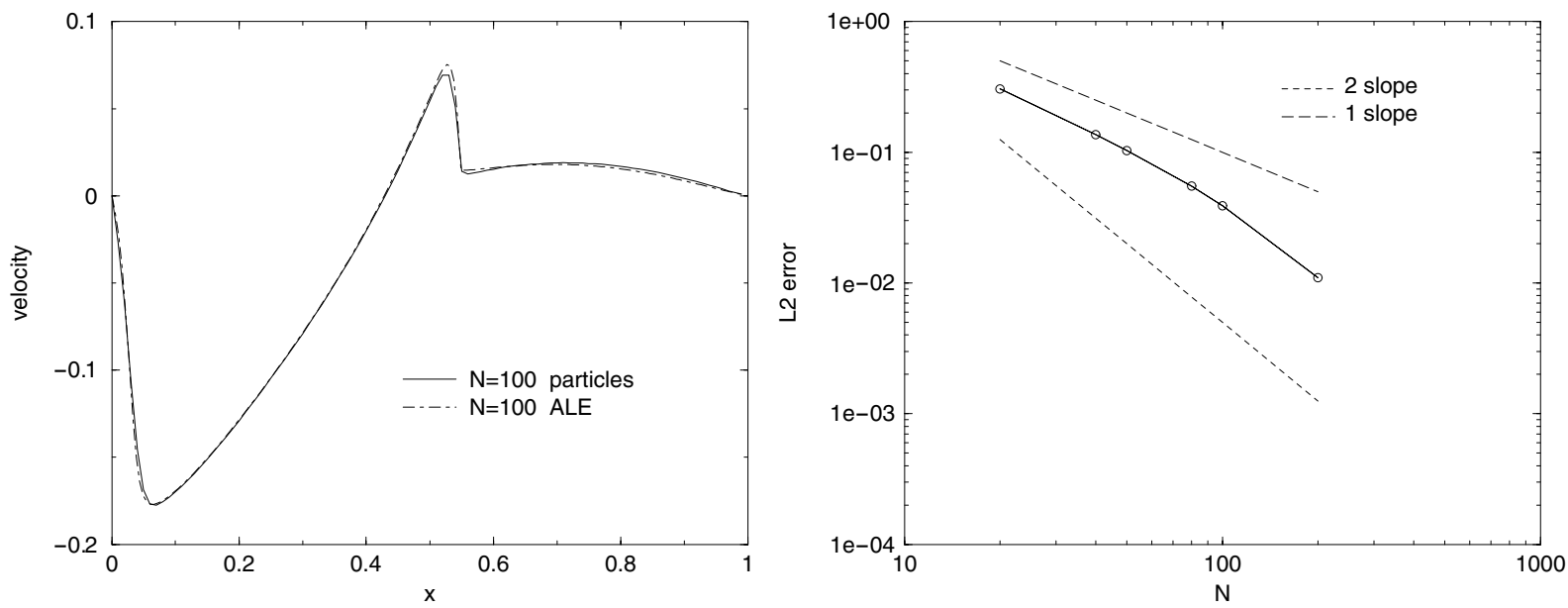

Fig. 3. Fluid-structure interaction: comparisons of PIC and ALE methods (left picture) and $L^{2}$ error curve for the PIC method (right picture).

\section{Conclusion}

Particle methods provide elegant tools to deal with interface problems in systems driven by complex physics. Their robustness and flexibility makes them appealing alternatives to ALE type methods, appropriate to handle strongly nonlinear behavior.

\section{References}

[1] Daru V, Tenaud C. Evaluation of TVD high resolution schemes for unsteady viscous shocked flows. Comput Fluids 2001;30(1):89-113.
[2] Cottet GH, Michaux B, Ossia S, Vanderlinden G. A comparison of spectral and vortex methods in three-dimensional incompressible flows. J Comp Phys 2002;175:702-712.

[3] Chang YC, Hou TY, Merriman B, Osher S. Eulerian capturing methods based on a level set formulation for incompressible fluid interfaces. J Comp Phys 1996;124:449464.

[4] Cottet GH. A particle model for fluid-structure interaction. C R Acad Sci Paris 2002;3:833-838. 